\title{
CZT camera: moving beyond classical CAD detection?
}

\author{
Thomas Hellmut Schindler
}

Published online: 24 March 2015

(C) Springer-Verlag Berlin Heidelberg 2015

In this issue of the European Journal of Nuclear Medicine and Molecular Imaging, Gimelli et al. [1] report that stressinduced left ventricular diastolic impairment, as reflected by the percent stress-to-rest peak filling rate (PFR) ratio, was paralleled by increasing numbers of coronary artery disease (CAD) vessels with stenosis $\geq 70 \%$. Conversely, the manifestation of stress-related systolic or contractile dysfunction was confined to patients with multivessel CAD. Such a novel observation may suggest indeed that stress-induced impairment of diastolic function of the left ventricle may reflect a more sensitive parameter to better identify and characterize the effects of ischemic burden imposed on the left ventricle than conventional assessment of post-stress systolic function. These observations are also unique as the myocardial perfusion exam was performed with a state-of-the art cadmium zinc telluride (CZT) camera that holds strong promise for determining myocardial perfusion and function with a fast acquisition time of about $7 \mathrm{~min}$ and low dose of ${ }^{99 \mathrm{~m}} \mathrm{Tc}$ injection for a single-day protocol (stress 185-222 MBq and rest 370$444 \mathrm{MBq}$ ), while maintaining excellent image quality [2-4].

The assessment of cardiac function with gated single photon emission computed tomography (SPECT) commonly aids in the identification of attenuation artifact on scintigraphic perfusion images and, thereby, substantially reduces false-

\footnotetext{
T. H. Schindler $(\square)$

Division of Nuclear Medicine, Cardiovascular Nuclear Medicine, Department of Radiology and Radiological Science SOM, JHOC, Johns Hopkins University, School of Medicine, 3225, $601 \mathrm{~N}$. Caroline Street, Baltimore, MD 21287, USA

e-mail: tschind3@jhmi.edu
}

positive findings. This again leads to an increase in specificity and thus diagnostic accuracy in CAD detection with conventional SPECT perfusion imaging [5]. With the advent of SPECT/CT cameras, the role of gated SPECT to identify attenuation artifacts has virtually disappeared due to the excellent attenuation correction with CT. This enables stress-only imaging in the majority of patients with normal perfusion findings that also confers an excellent cardiovascular outcome $[6,7]$. Since in the current investigation [1] no attenuation correction of CZT images was performed, myocardial perfusion imaging in conjunction with wall motion analysis most likely was needed in a certain number of patients to avoid false-positive findings due to attenuation artifacts, in particular in obese individuals and women [8]. Given the increased use of SPECT/CT cameras, post-stress gated SPECT is predominantly used to identify myocardial wall motion abnormalities as a sign of prolonged postischemic stunning. Stress-induced stunning is a marker of severe ischemia related to one-vessel or multivessel high-grade epicardial narrowing, respectively. Notably, post-stress global systolic dysfunction has a higher sensitivity for the detection of severe and multivessel CAD as compared to perfusion defects alone. This may be related, at least in part, to the normalization of radiotracer uptake on SPECT or SPECT/CT images. Perfusion images are normalized to the area of highest radiotracer uptake within the myocardium so that in the presence of stress-induced diffuse ischemia underlying multivessel CAD only the most severely hypoperfused region may reveal a perfusion defect. Given the presence of advanced and severe multivessel CAD, the relative distribution of radiotracer uptake or perfusion of the left ventricle may be homogeneously reduced or with only a mild visually discernible regional perfusion defect during stress 
testing. For example, Lima et al. [9] investigated the added value of combined perfusion and function assessment over perfusion alone gated SPECT for the detection of severe three-vessel CAD in 143 patients. Only in $10 \%$ of these patients with angiographically proven severe three-vessel CAD stress-related regional perfusion defects could be detected. When adding wall motion abnormalities on post-stress gated SPECT to myocardial perfusion imaging, the detection of three-vessel disease increased but only to $25 \%$. In another seminal investigation, Berman et al. [10] investigated 101 CAD patients with left main stenosis $\geq 50 \%$ with gated exercise or adenosine stress ${ }^{99 \mathrm{~m}} \mathrm{Tc}$-sestamibi SPECT myocardial perfusion imaging. When analyzing myocardial perfusion visually and semiquantitatively, a high-risk criterion with moderate to severe perfusion defects ( $>10 \%$ myocardium at stress) was observed in 56 and $59 \%$ of patients, respectively. On the other hand, there was no significant stress-related perfusion defect ( $\geq 5 \%$ myocardium) in $13-15 \%$ of patients. The evaluation of both abnormal perfusion and wall motion on poststress gated SPECT, however, yielded an $83 \%$ diagnostic accuracy for the detection of high-risk individuals with flowlimiting left main lesion during higher coronary flows. Current findings of myocardial perfusion and function imaging with a CZT camera also confirm that post-stress global systolic dysfunction signifies advanced multivessel CAD [1]. Although a fast imaging protocol was used, stress image acquisition was performed only $10-15 \mathrm{~min}$ after radiotracer injection. Thus, it is equally possible that post-stress systolic dysfunction or myocardial stunning in patients with one-vessel or two-vessel disease had actually resolved within the time frame of 10-15 min, leaving only stress-related regional perfusion defects to suggest hemodynamically obstructive CAD. Conversely, multivessel disease with epicardial narrowing $\geq 70 \%$ is likely to have caused diffuse and severe ischemia leading to a more long-lasting and global myocardial stunning still present 10-15 min after stress testing. Overall, it would be desirable to perform wall motion analysis during "peak" stress as it has been reported more recently for ${ }^{82} \mathrm{Rb}$ positron emission tomography (PET)/CT studies [11]. The value of vasodilator left ventricular ejection fraction (LVEF) reserve (stress LVEFrest LVEF) in analyzing the magnitude of myocardium at risk and the anatomic extent of underlying severe CAD was evaluated. A decrease in LVEF reserve $-5 \%$, more commonly used in routine clinical practice, yielded a sensitivity, specificity, and positive and negative predictive values of $59 \%, 94 \%$, $77 \%$, and $87 \%$, respectively. Thus, a normal LVEF reserve, as determined with gated PET during peak stress and at rest, widely rules out left main and/or severe three-vessel CAD disease. A similar analysis evaluating the diagnostic accuracy of stress-rest CZT assessment of perfusion and function in the detection of left main and/or severe multivessel disease is still eagerly awaited. Such an investigation with wall motion assessment by CZT imaging should be preferentially performed at peak vasodilator stress, like for gated PET [11], in order to avoid a "diagnostic gap" with recovery of ischemia-induced regional or global systolic dysfunction 10-15 min after stress testing. For example, when performing $82 \mathrm{Rb}$ gated PET in patients with suspected CAD, an abnormal LVEF reserve (less than $+5 \%$ ) was appreciated in 30,62 , and $94 \%$ in patients with one-vessel, two-vessel, and left main/three-vessel CAD, respectively. Thus, it appears that in current CZT imaging study reduction in LVEF reserve or stress-induced wall motion abnormalities due to one-vessel or, at least in part, due to two-vessel disease may have been missed due to post-stress imaging at 10-15 min instead of peak stress imaging with gated PET [11]. As it was observed in the current investigation with the use of the CZT camera, the PFR ratio progressively decreased with increasing numbers of narrowed $C A D$ vessels. Such observations may accord with the functional hierarchy among different ischemic manifestations such as sequential alterations of diastolic relaxation and systolic contraction pattern, subsequently followed by electrocardiographic and symptomatic manifestation [12]. The decrease in PFR ratio, however, may also be seen in patients with left ventricular hypertrophy predominantly in patients with arterial hypertension but also in individuals with diabetes mellitus and/or advanced obesity. Thus, although a decrease in PFR ratio may afford an earlier identification and characterization of CADrelated ischemic burden on the left ventricle, it may also be appreciated as a nonspecific relaxation parameter in the presence of left ventricular hypertrophy that necessitates the application and interpretation of the PFR ratio in the proper clinical context with underlying coronary anatomy and cardiovascular risk factors. How current novel observations of Gimelli et al. [1] with the CZT camera in the assessment of myocardial perfusion and function for CAD detection will find its way into clinical practice remains to be seen. Nevertheless, the comprehensive assessment of cardiac perfusion and function with CZT technology with short image acquisition is another enrichment and advancement in the field of scintigraphic myocardial perfusion imaging in an era demanding reduced costs, examination time, and radiation exposure.

Conflicts of interest None.

\section{References}

1. Gimelli A, Liga R, Giorgetti A, Kusch A, Pasanisi EM, Marzullo P. Relationships between myocardial perfusion abnormalities and poststress left ventricular functional impairment on cadmium-zinctelluride imaging. Eur J Nucl Med Mol Imaging 2015. doi:10.1007/ s00259-015-3008-7.

2. Giorgetti A, Masci PG, Marras G, Rustamova YK, Gimelli A, Genovesi D, et al. Gated SPECT evaluation of left ventricular function using a CZT camera and a fast low-dose clinical protocol: 
comparison to cardiac magnetic resonance imaging. Eur J Nucl Med Mol Imaging 2013;40:1869-75.

3. Gimelli A, Liga R, Pasanisi EM, Giorgetti A, Marras G, Favilli B, et al. Evaluation of left ventricular diastolic function with a dedicated cadmium-zinc-telluride cardiac camera: comparison with Doppler echocardiography. Eur Heart J Cardiovasc Imaging 2014;15:972-9.

4. Gimelli A, Liga R, Giorgetti A, Casagranda M, Marzullo P. Relationship between myocardial perfusion abnormalities and contractile impairment in anginal patients. J Nucl Cardiol 2014;21:118190.

5. Mut F, Giubbini R, Vitola J, Lusa L, Sobic-Saranovic D, Peix A, et al. Detection of post-exercise stunning by early gated SPECT myocardial perfusion imaging: results from the IAEA multi-center study. J Nucl Cardiol 2014;21:1168-76.

6. Chang SM, Nabi F, Xu J, Raza U, Mahmarian JJ. Normal stress-only versus standard stress/rest myocardial perfusion imaging: similar patient mortality with reduced radiation exposure. J Am Coll Cardiol 2010;55:221-30.

7. Duvall WL, Savino JA, Levine EJ, Hermann LK, Croft LB, Henzlova MJ. Prospective evaluation of a new protocol for the provisional use of perfusion imaging with exercise stress testing. Eur J Nucl Med Mol Imaging 2015;42:305-16.

8. Valenta I, Schindler TH. (82)Rb PET/CT: entering a new area of myocardial perfusion imaging? Eur J Nucl Med Mol Imaging 2012;39:1231-2.

9. Lima RS, Watson DD, Goode AR, Siadaty MS, Ragosta M, Beller $\mathrm{GA}$, et al. Incremental value of combined perfusion and function over perfusion alone by gated SPECT myocardial perfusion imaging for detection of severe three-vessel coronary artery disease. J Am Coll Cardiol 2003;42:64-70.

10. Berman DS, Kang X, Slomka PJ, Gerlach J, de Yang L, Hayes SW, et al. Underestimation of extent of ischemia by gated SPECT myocardial perfusion imaging in patients with left main coronary artery disease. J Nucl Cardiol 2007;14:521-8.

11. Dorbala S, Vangala D, Sampson U, Limaye A, Kwong R, Di Carli MF. Value of vasodilator left ventricular ejection fraction reserve in evaluating the magnitude of myocardium at risk and the extent of angiographic coronary artery disease: a $82 \mathrm{Rb}$ PET/CT study. J Nucl Med 2007;48:349-58.

12. Detry JM. The pathophysiology of myocardial ischaemia. Eur Heart J 1996;17(Suppl G):48-52. 